Die in der Rubrik * stehende Zahl bezieht sich auf die Nummern des vorangehenden Verzeichnisses, die übrigen Sterne wurden für den Anfang des Beobachtungsjahres folgendermassen angenommen:

a) $22^{\mathrm{h}} 22^{\mathrm{m}} 52^{\circ} 96+33^{\circ} \mathrm{ŏ}^{\prime} 23^{\prime \prime} 0$ Leidner Annalen, 4. Band.

b) 23.33.24.17 - 14.5்.6.5 w' Aquarii, Sev. y. C. 1977 New Sev. y. C. 2707.

c) 23.16.30.68 - 20.46.17.8 b' Aquarii, New Sev. y. C. 2677 Sev. y. C. 1957 Yarn. 10305.

d) $1.35 .41 .50+10.43 .35 .8$ Rümk. II 839. Astr. N. 32, 217. Lam. 232.

e) $1.13 .11 .33+10.18 .40 .4$ Sjell. 415 .

f) $2.26 .10 .05+14.29 .19 .329$ Arietis.

g) 8.40.53.64 + 18.30.9.0 Astr. N. 29, 341 .

h) $9.59 .6 .64+11.29 .30 .2$ Weisse 1244 .

i) 10.22.56.94 + 12.48.38.2 Mittel aus $\mathrm{Sj}$. 3841, Yarnall u. 63 des vorrang. Verz.

k) $10.0 .2 .74+15.2 .14 .8$ B.D., + $15^{0} 2165$, Astr. N. 58, 234.

l) $9.56 .6 .63+15.23 .54 .5$ Astr. N. 88, 245 .

m) $11.8 .22 .59+7.32 .59 .3$ Lam. 532, Astr. N. 43,$233 ; 44,327$.

Pola, April 1878.

J. Palisa.

\title{
Berechnung der Elemente des Cometen III 1877.
}

Der Comet wurde unabhängig entdeckt am 11. April 1877 von Herrn Lewis Swift in Rochester U. S. NewYork, am 14. April von Herrn Borrelly in Marseille, endlich am 16. April von Herrn Block in Odessa.

Aus den drei folgenden vollstandigen Beobachtungen, welche auf der Sternwarte zu Leipzig angestellt waren, wurde von mir zuerst eine parabolische Bahn gerechnet.

Mittl. Zeit Leipzig a app. o app.

April $15 \quad 11^{\mathrm{l}} 48^{\mathrm{m}} 8^{\mathrm{s}} \quad 1^{\mathrm{h}} 14^{\mathrm{m}} 2^{\mathrm{s}} 47+55^{0} \overline{5}^{\prime} 13^{\prime \prime} 3$

April 26 9.23.9 3.13.25.21 9 + 61.21 .55 .6

Ilai $T 10.43 .20$ 5.36.45.67 + 58.23.19.7

Die Elemente sind:

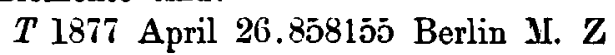

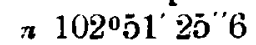

$\Omega 346.4 .12 .9$

i 77.10 .8 .81

$\log q \quad 0.004008$

Mit einer aus diesen Elementen gerechneten Ephemeride ergaben sich unter Berücksichtigung der Aberration und Parallaxe die folgenden Differenzen, als Resultat der Vergleichung der Beobachtungen mit der Ephemeride und im Sinne B.-R. :

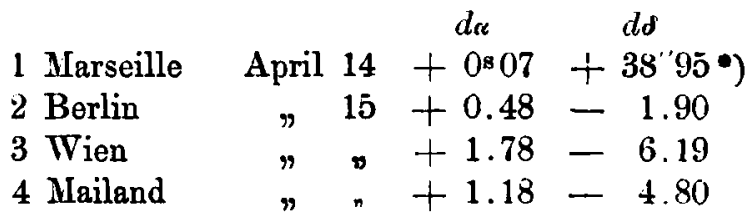

*) Die Beobachtung in $\delta \mathrm{zu}$ Marseille wurde als fehlerhaft ausgeschlossen und die Beobachtung Nr. 46 wurde in $c$ um $30^{\circ}$ corrigirt.

\begin{tabular}{|c|c|c|c|c|c|}
\hline & & & $d \omega$ & & $d \delta$ \\
\hline 5 Strassburg & April & 15 & $+1^{8} 22$ & 一 & 1 '53 \\
\hline 6 Leipzig & $r$ & & +0.13 & - & 0.60 \\
\hline 7 Leipzig & & ") & +0.57 & + & 7.69 \\
\hline 8 Wien & $\pi$ & $"$ & +0.09 & - & 7.92 \\
\hline 9 Kiel & & $n$ & $\ldots \ldots$ & + & 0.11 \\
\hline 10 Hamburg & $n$ & n & +0.64 & - & 3.66 \\
\hline $11 \mathrm{Kiel}$ & $\eta$ & n & +2.29 & . & $\ldots \ldots$ \\
\hline 2 Wien & n & 16 & $+0 . \overline{58}$ & 一 & 0.15 \\
\hline 13 Hamburg & $\pi$ & $n$ & -1.04 & - & 3.34 \\
\hline 4 Mannheim & $m$ & 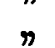 & +0.64 & - & 0.27 \\
\hline 15 Kiel & $n$ & $n$ & $\ldots \ldots$ & $-t$ & 13.31 \\
\hline 6 Ode & $n$ & $\eta$ & +2.10 & + & 6.97 \\
\hline 17 Leip? & n & n & +0.94 & - & 1.11 \\
\hline $18 \mathrm{Kiel}$ & $n$ & " & +0.64 & . & \\
\hline 19 Leipzig & & $n$ & +0.48 & + & 1.15 \\
\hline $20 \mathrm{Kiel}$ & & & -3.25 & + & 3.81 \\
\hline 21 . Odessa & & $"$ & +2.58 & + & 4.11 \\
\hline 22 Odessa & $n$ & 17 & +2.47 & + & 2.58 \\
\hline 23 Leipzig & $n$ & $\eta$ & -0.19 & - & 2.80 \\
\hline 24 Hamburg & " & $n$ & +0.05 & 一 & 1.16 \\
\hline $25 \mathrm{Kiel}$ & $n$ & 18 & -1.38 & - & 8.77 \\
\hline $26 \mathrm{Kiel}$ & $n$ & $\eta$ & +1.61 & + & 2.56 \\
\hline 27 Odessa & & 19 & +2.50 & - & 14.94 \\
\hline 28 Mailand & $\eta$ & $\pi$ & -0.02 & + & 2.84 \\
\hline 29 Christiania & $\eta$ & 20 & +0.16 & - & 4.11 \\
\hline 30 Hamburg & $\eta$ & 川 & -1.51 & - & 5.67 \\
\hline 31 Hamburg & $n$ & 21 & -0.38 & + & 5.31 \\
\hline 32 Hamburg & $\eta$ & 22 & -0.22 & - & 1.93 \\
\hline
\end{tabular}




33 Mailand
34 Christiania
35 Leipzig
36 Leipzig
37 Christiania
38 Kiel
39 Kiel
40 Christiania
41 Kiel
42 Leipzig
43 Kiel
44 Christiania
45 Athen
46 Kiel
47 Leipzig
48 Christiania
49 Kiel
50 Leipzig
51 Leipzig
52 Kiel
53 Kiel
54 Leipzig
55 Leipzig
56 Athen

\begin{tabular}{|c|c|c|c|c|}
\hline \multirow{2}{*}{\multicolumn{2}{|c|}{ il 24}} & $d \alpha$ & & $d d$ \\
\hline & & +1818 & - & $4^{\prime \prime} 22$ \\
\hline & & +0.19 & - & 2.17 \\
\hline & & +1.90 & 一 & 5.52 \\
\hline & 26 & -0.94 & - & 1.81 \\
\hline & 29 & +0.30 & + & 3.04 \\
\hline & & +1.37 & - & 6.53 \\
\hline Mai & 1 & -3.89 & + & 5. 26 \\
\hline$n$ & 2 & -0.74 & + & 3.42 \\
\hline r & 3 & +2.41 & - & 2.11 \\
\hline & & -0.12 & - & 5.76 \\
\hline 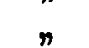 & 4 & +127 & + & 13.79 \\
\hline . & . & -1.34 & - & 3.58 \\
\hline 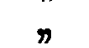 & 6 & +2.13 & + & 12.09 \\
\hline & 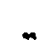 & $\left.+32.46^{*}\right)$ & - & อ. 70 \\
\hline & $n$ & -0.83 & - & 208 \\
\hline & & -0.13 & + & 3.60 \\
\hline & 7 & +1.72 & + & 6.10 \\
\hline & & -0.01 & - & 0.24 \\
\hline & 8 & -1.26 & - & 4.66 \\
\hline & $\pi$ & $+1.6 x$ & + & 1054 \\
\hline & 9 & +1.37 & - & 1.41 \\
\hline & 10 & -0.96 & + & 0.78 \\
\hline & 11 & -078 & - & 416 \\
\hline & 12 & -0.33 & - & 615 \\
\hline
\end{tabular}

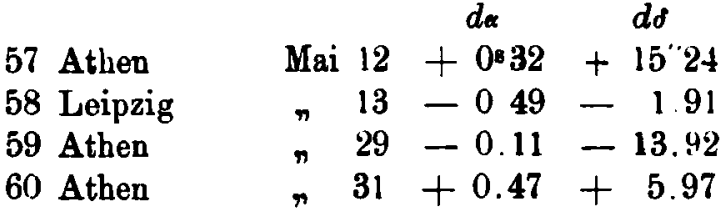

Aus den obigen Differenzen ergaben sich die folgenden Correctionen der Ephemeride tür 5 Normalörter : $\cos \delta d \alpha$ Zahl d. Beob. do Zahl d. B.-R. B. - $R$ Beob.

$\begin{array}{rcrrrrr}\text { I } & \text { April } & 16.5 & +4^{\prime \prime 764} & 26 & -0.926 & 25 \\ \text { II } & 7 & 22.5 & +0.637 & 8 & -2.600 & 8 \\ \text { III } & \text { Mai } & 2.5 & -1.598 & 8 & +0.415 & 8 \\ \text { IV } & 7 & 85 & -0.032 & 14 & +0.660 & 14 \\ \text { V } & { }^{\prime} & 30.5 & +2.171 & 2 & -4.040 & 2\end{array}$

Nach Anbringung von $d a$ und $d \delta$ an die Ephemeride, erhält man als Normalörter, bezogen auf mittl. Aequat. 1877 :

$\begin{array}{rrr}\text { I } & 20^{\circ} 33^{\prime} 16^{\prime \prime} 26 & +56^{\circ} 34^{\prime} 39^{\prime \prime} 4 \prime \prime \\ \text { II } & 36.14 .37 .15 & +60.7 .17 .11 \\ \text { III } & 68.5312 .52 & +60.55411 .21 \\ \text { IV } & 87.7 \quad 8.18 & +57.37 .53 .81 \\ \text { V } & 123.6 .37 .27 & +3554.39 .83\end{array}$

Für diese fünf Normalörter wurden darauf die folgenden Bedingungsgleichungen berechnet.

in $\alpha$

Bedingungsgleichungen.

$[0.97248 n]\left(10^{8} d T\right)+[0.35194]\left(10^{5} d q\right)+[028515] d n+[0.31694 n] d \Omega+[0.41786 n] d i+[1.33909 n]=0$

$[0.89791 n] n+[1.32865] n+[0.20896] n+[0.23546 n] n+[0.19506 n] n+[0.25608 n]=0$

$[0.92700 n] n+[0.39751] n+\left[\begin{array}{ll}0 & 22135]\end{array}\right]+[0.15+20 n] n+[9.29184 n] n+[0.59279]=0$

$[1.01169 n] \%+[0.51919] \pi+[0.28763] "+[0.07784 n] n+[9.54200] n+[9.01737]=0$

$[0.11099 n] "+[9.96143[n+[9.17082] "+[9.58364] n+[9.29605] "+[0.3365 i n]=0$

in $d$

$[0.90931 n] \eta+[1.14169] n+[0.29581] \eta+[0.10588 n] \eta+[0.07520] n+[0.62249]=0$

$[0.54699] "+[0.89394] "+[9.90116] n+[9.31218 n] n+[0.04856] n+[0866 \mathbf{b} 2]=0$

$[0.18922] n+[0.75857] n+[9.59279 n] n+[0.04550] n+[0.07979] n+[0.00712 n]=0$

$[0.75443] n+[0.87964] " n+[0.12971 n] n+[0.35237] n+[0.13120] n+[0.33014 n]=0$

$[0.44152] "+[0.50699] "+[9.86710 n] "+[9.87506] "+[884504] "+[0.60638]=0$

Die eingeklammerten Zahlen sind die Logarithmen der Coeffieienten für die beigesetzten Elementenanderungen. Unter $n$ sind die ubrigbleibenden Fehler der Ephemeride aufgefuhrt, selbstverständlich die $d_{\alpha}$ mit $\cos \&$ multiplicirt. Den einzelnen Bedingungsgleichungen sind auch schon der Reihe nach die Gewichte 21, 8, 6, $10^{1 / 2}, 1 ; 20^{1} / 2,8,6,10^{1} / \mathrm{s}, 1$, gegeben, d. h. sie sind mit den Quadratwurzeln aus den Gewichten multiplicirt. Es ergaben sich, nachdem diese Bedingungsgleichungen nach der Methode der kleinsten Quadrate aufgelöst waren, als Endgleichungen:

$$
\begin{aligned}
& +449.8155\left(10^{8} d T\right)-173.4538\left(10^{5} d q\right)-94.2172 d \pi+84.5741 d \Omega+28.3731 d i+125.3123=0 \\
& +314.7775 n+0.9465 n+23.6663 n+45.8528 n+110.1692=0 \\
& +0.2057 n+0.4839 n+0.0182 n+1.7460=0 \\
& +0.5876 \%+1.4546 n+4.3442=0 \\
& +2.4700+32.7430=0
\end{aligned}
$$


Die daraus gefundenen Elementencorrectionen sind:

$$
\begin{aligned}
d T & =+0.006584 \\
d \pi & =+52^{\prime \prime} 491 \\
d \delta & =+25.423 \\
d i & =-13.256 \\
d(\log q) & =-0.0000049
\end{aligned}
$$

nach deren Anbringung an die vorherghenden Elemente man als wahrscheinlichstes Elementensystem erhält:

$$
\begin{aligned}
T & =1877 \text { April } 26.864739 \text { M. B. Z } \\
\pi & =102^{\circ} 52^{\prime} 18^{\prime \prime}(99 \\
\Omega & =346 \quad 4.38 .32 \\
i & =7 \tau .9 .50 .55
\end{aligned}
$$$$
\log q=0.0040031
$$

Die Substitution der gefundenen Correctionen in die Bedingungs - Gleichungen, sowie die Vergleichung der Normalörter mit einer aus den wahrscheinlichsten Elementen berechneten Ephemeride, ergab folgende ubrig bleibende Fehler der Normalörter:

Bedingungs-Gleichungen

$$
\begin{aligned}
& d r \cos \delta \quad d \delta \\
& +0^{\circ} 34+0 \% 11 \\
& -1.00-0.74 \\
& -0.31+0.77 \\
& +0.74-0.03 \\
& -3.79-0.11
\end{aligned}
$$

Directe Rechnung

$$
\begin{aligned}
& d \boldsymbol{c o s} \delta \quad d \delta \\
& +0.33+0.0 x \\
& -1.00-078 \\
& -0.35+0.78 \\
& +0.76-0.05 \\
& -3.85-0.06
\end{aligned}
$$

Obwohl die übrig bleibenden Fehler sehr gering sind, also die Parabel den Beobachtungen sehr nahe genügt, habe ich doch noch rersucht eine Aenderung der Excentricitat der Bahn einzuführen und zu den vorhergehenden Bedingungsgleichungen kommen noch dio folgenden Glieder für $10^{4}$ de hinzu.

$$
\text { in } \alpha \text { in } \delta
$$

$$
\begin{array}{ll}
{[0.66555 n]} & {[0.59777 n]} \\
{[0.20038 n]} & {[9.84551 n]} \\
{[0.3: 050]} & {[9.56264 n]} \\
{[0.71061]} & {[0.43461 n]} \\
{[0.28533]} & {[0.51558 n]}
\end{array}
$$

Löset man die Bedingungsgleichungen von neuem nach der Methode der kleinsten Quadrate auf, so finden sich dei folgenden Elementencorrectionen:

$$
\begin{aligned}
d T & =+0.0101408 \\
d e & =+0.0003446 \\
d \pi & =+53.6291 \\
d \delta & =+16.9212 \\
d i & =-220932 \\
d(\log q) & =+00000238
\end{aligned}
$$

und damit die Elemente:

$$
\begin{aligned}
T & =1877 \text { April } 26.868296 \\
e & =1.0003446 \\
\pi & =102^{\circ} 52^{\prime} 19^{\circ} 23 \\
8 & =346.4 .29 .82 \\
i & =779.46 .72 \\
\log q & =0.06403 \% .
\end{aligned}
$$

Die übrigbleibenden Fehler in diesem Falle sind:

$$
\begin{gathered}
d \alpha \cos \delta \quad d \delta \\
+0.32+0.15 \\
-0.99-0.84 \\
-0.33+0.70 \\
+0.76-0.01 \\
-3.62-0.03
\end{gathered}
$$

Wahrend bei der Parabel [n. n. 5] $=39^{\prime \prime} 07$ war, ist bei dieser hyperbolischen Bahn $[n . n .6]=38^{\prime \prime} 99$, also so wenig geringer, dass die Parabel den Normalörtern fast ebenso genau genügt, als die hyperbolische Bahn. Um schliesslich noch die unsicherheit der Elemente zu finden, berechnete ich die Gewichte der einzelnen Elemente, und finde als wahrscheinliche Fehler der Elemente:

$$
\text { Wahrscheinlicher Fehler von } \begin{aligned}
d T & = \pm 0.0(1) 3534 \\
d e & = \pm 0 .(000353 \\
d(\log q) & = \pm 0.0010028 \\
d \pi & = \pm 2.0 \\
d \delta & = \pm 8.8 \\
d i & = \pm 88
\end{aligned}
$$

Leipzig d. 30. Mai 1878.

\section{J. Walter Nichol.}

\section{Beobachtungelı der Sternwarte Kremsmünster im Jahre 1877.}

(Fortsetzung) Meridiankreis.

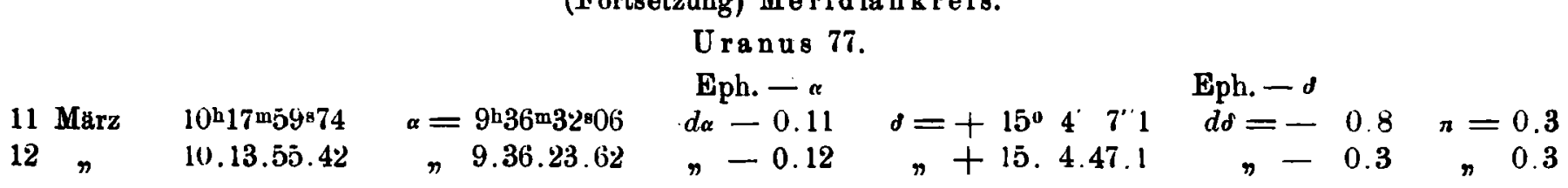

